\title{
Características en estrategias de aprendizaje en matemáticas por alumnos mexicanos de bachillerato
}

Characteristics in the use of mathematics learning strategies by Mexican senior high school students

Características em estratégias de aprendizagem em matemática por alunos mexicanos de bacharelado

DOI: https://doi.org/10.18861/cied.2021.12.1.3012

\section{Prócoro Omar Butrón Zamora}

Benemerita Universidad Autonoma de Puebla

México

omar_21063@hotmail.com

ORCID: 0000-0001-7221-265X

\section{José Gabriel Sánchez Ruiz}

Facultad de Estudios Superiores Zaragoza, Universidad Nacional Autonoma de Mexico

Benemerita Universidad Autonoma de Puebla

México

josegsr@unam.mx

ORCID: 0000-0002-4306-1431

Fecha de recibido: 16/10/2020

Fecha de aprobado: 16/02/2021

Cómo citar: Butrón Zamora, P. O., \& Sánchez Ruiz, J. G. (2021). Caracteristicas en estrategias de aprendizaje en matematicas por alumnos mexicanos de bachillerato. Cuadernos de Investigación Educativa, 12(1). https://doi. org/10.18861/cied.2021.12.1.3012

\section{Resumen}

Las estrategias de aprendizaje (cognitivas, metacognitivas y estrategias relacionadas con el uso de recursos) están estrechamente vinculadas con el rendimiento académico del alumno posibilitando mejorar el aprendizaje. Las estrategias de aprendizaje se han considerado herramientas fundamentales en los procesos de enseñanza-aprendizaje en la totalidad de las áreas del conocimiento, entre ellas en matematicas. El objetivo del presente estudio es detectar las características en el empleo de estrategias de aprendizaje en matemáticas en alumnos de bachillerato y posibles diferencias en función del curso académico y del sexo. Se aplicó el cuestionario LIST (Estrategias de aprendizaje en la universidad) a 154 estudiantes 91 mujeres (59,1\%) y 63 varones $(40,9 \%)$. Se encontro una confiabilidad muy aceptable del instrumento $(\alpha=0,94)$. Por un lado el grupo de alumnos del segundo semestre tiene tendencia a recurrir más a las estrategias evaluadas en comparación con los demás grupos. Por otro lado, las estudiantes tienen tendencia a gestionar mejor los saberes matemáticos, a tene un ambiente de aprendizaje adecuado y a la búsqueda de frecuencia que los varones.

Palabras clave: estrategias de aprendizaje, estrategias cognitivas, estrategias metacognitivas, estrategias relacionadas con los recursos, matemáticas, bachillerato, cuestionario LIST.

\section{Abstract}

Learning strategies (cognitive and metacognitive strategies as well as those related to the use of resources) are closely linked to the student's academic performance, making possible to improve learning. Learning strategies have been considered fundamental tools in teaching-learning processes in all areas of knowledge, including mathematics. The goa of the present research is to detect the characteristics in the use of tearning strategles in mathematics in high school students as well as any difference that may arise according to academic year and gender. The LIST questionnarie (Learning strategies at University) was applied to 154 students. 91 femate students $(59,1 \%)$ and 63 mate students $(40.9 \%)$. A very acceptable reliability of the instrument was found $(\alpha=0,94)$. On one hand, the group of students in the second semester has a tendency to make more use of the strategles evaluated, as compared to the other groups. On the other hand, female students tend to manage mathematical knowledge in a better way, to have a sultable learning environment and to search for information in different sources when facing difficulties, more often than male students.

Keywords: learning strategies, cognitive strategies, metacognitive strategies, resourcerelated strategies, mathematics, high school, LIST questionnaire.

\section{Resumo}

As estrategias de aprenaizagem (cognitivas, metacognitivas e estrateglas relacionadas com o uso de recursos) estáo estreltamente vinculadas ao desempenno academico do aluno possibittando melhorar o aprendizado. As estrategias de aprendizagem foram consideradas ferramentas fundamentais nos processos de ensino-aprendizagem em odas as areas do conhecinento, entre elas na matemática. Oobjectivo do presente estudo éctectar as caracteristicas no ennprego de estrategias de aprendizagem em matematica en alunos do ensino secundario possiteis differenças em funçáo do ano acadènico do sexo. Aplicou-se 0 questionário LIST (Estrategias de aprendizagem na Universidade)

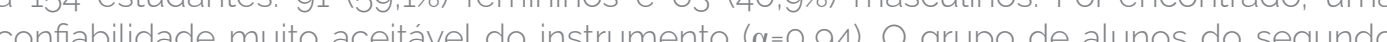
confiabilidade muito aceitavel do instrumento $(\alpha=0,94)$. O grupo de alunos do segundo os outros ten tendencia a recorrer mals as estrateglas avaltadas ent comparaçăo com os saberes mastemáticos a ter um estudantes femininos tem tendêncla a geril melhor os saberes matematicos, a ter um ambiente de aprendizagen adequado e à busca de Palavras-chave: estratégias de aprendizagem, estratégias cognitivas, estratégias metacognitivas, estrateglas relacionadas com os recursos, matemáticas, bacharelado, questionário LIST. 


\section{Introducción}

Las estrategias de aprendizaje (EA), entre otros aspectos, constituyen una herramienta esencial en el aprendizaje y enseñanza de las matemáticas, afectan las metas de un modelo educativo e inciden en el tipo de aprendizaje que se pretende lograr, por lo que resulta necesaria su correcta identificación (GascoTxabarri, Ros y Goñi, 2017).

En los últimos años las EA han venido conformando uno de los dominios de conocimiento más abordados por la psicología de la educación, no solo por su posibilidad de vinculación con diferentes constructos teóricos sino también por la importancia inherente que conlleva su utilización. Las EA están estrechamente vinculadas con el rendimiento académico del alumno y permiten mejorar el aprendizaje. Estas son razones válidas para que muchos investigadores intenten conocer mejor estos constructos que tanto interés despiertan, e indaguen en todas sus posibilidades en el ámbito de la educación matemática (Calderón y Chiecher, 2012)

Para Beltrán (2003) las EA son herramientas del pensamiento que el alumno pone en acción ante un contenido. Las mismas generan infinidad de posibilidades de aprendizaje y potencian las acciones del pensamiento a límites insospechados. Una cuestión clave en el estudio de las EA reside en precisar el número y naturaleza de estas, dada la variabilidad entre las diferentes propuestas al respecto y sus correspondientes instrumentos de medida (Beltrán, 1993). En este sentido, Gasco-Txabarri et al. (2017) refieren que, para conocer el empleo de las EA en el aprendizaje de las matemáticas, resulta imprescindible disponer de cuestionarios que permitan una medición fiable del constructo que se pretende medir, por ejemplo, el Cuestionario de Estrategias de Aprendizaje en Matemáticas (CEAMA de Gasco-Txabarri et al., 2017).

La investigación en educación matemática en el nivel medio superior puede ser un campo de investigación interesante que podría conducir a resultados útiles que los docentes de todos los niveles educativos podrían aplicar a su enseñanza. En lo referente a la educación, en ciencia, tecnología, ingeniería y matemáticas (STEM), el paso de la educación de nivel medio superior al nivel superior se considera problemático, lo que es particularmente cierto con respecto a los desafios que se presentan a los estudiantes cuando se enfrentan a las matemáticas universitarias, según Kaldo y Õun (2019).

\section{Antecedentes}

Las EA se han estudiado en diversos trabajos. Kaldo y Õun (2019), por ejemplo, analizaron las EA de los estudiantes universitarios estonios de primer año en matemáticas. Los datos fueron recolectados durante dos años en 440 estudiantes universitarios de diferentes disciplinas. El objetivo de esta investigación fue examinar el Cuestionario LIST de Estrategias de Aprendizaje en Matemáticas de 69 items, por primera vez, en estudiantes universitarios de Estonia.

En otro estudio Kecici y Aydin (2019) trabajaron con 754 estudiantes de distintas áreas de la educación de una universidad estatal de Turquía. En este estudio se adaptó el cuestionario LIST, traducido al turco por Boerner, Seeber, Keller y Beinborn (2005) para definir las EA de los estudiantes universitarios. Se realizaron análisis de validez y fiabilidad a esta versión teniendo en cuenta la forma original. Griese, Lehmann y Roesken-Winter (2015) aplicaron el cuestionario LIST a 2374 estudiantes de STEM de diferentes cursos de ingeniería de primer semestre en la Ruhr-Universität Bochum (Universidad Ruhr de Bochum, en Alemania), 
predominantemente del sexo masculino. El objetivo fue examinar el cuestionario LIST de 69 items para reducirlo manteniendo su estructura de factores y, por lo tanto, su potencial para describir el comportamiento de aprendizaje asi como para identificar cambios significativos en el mismo.

En otro trabajo, Gómez-Chacón, Griese, Rösken-Winter y González-Guillén (2015) exploraron mediante el cuestionario LIST en dos muestras de estudiantes de ingeniería: 113 españoles y 159 alemanes. Los resultados muestran que ambos grupos de estudiantes difieren con respecto a su puntuación en las escalas organización, elaboración, repetición y metacognición. Las diferencias en las EA entre los dos paises se pueden concentrar en el hecho de que los estudiantes alemanes de ingeniería mostraron más variación en las estrategias usadas y, a menudo, obtuvieron calificaciones más bajas en matemáticas, lo que significa que los estudiantes españoles tendieron a expresar el comportamiento deseado, es decir, actividades de aprendizaje diligentes.

Gasco-Txabarri (2016) se enfocó en el estudio de posibles diferencias en el empleo de las estrategias de aprendizaje en función del curso académico. Para ello recogió datos de 565 estudiantes de segundo, tercero y cuarto curso de Educación Secundaria Obligatoria (ESO). Utilizó una adaptación del Cuestionario de Motivación y Estrategias de Aprendizaje (Motivated Strategies for Learning Questionnaire - MSLQ) de Pintrich, Smith, García y McKeachie (1993). Los resultados indican un aumento en el empleo de la mayoría de las EA a medida que el curso sube de grado, con la única excepción de la estrategia de repetición aunque no en todas sus dimensiones.

Gasco-Txabarri et al. (2017) analizaron la estructura factorial de la versión castellana del Cuestionario de Estrategias de Aprendizaje para las Matemáticas (CEAMA) que mide el empleo de estas estrategias en dicha asignatura. Los hallazgos obtenidos están relacionados con tres cuestiones: la organización factorial encontrada -que tuvo una estructura heptaescalar-, el tipo de estrategias metacognitivas observadas - esencialmente de planificación y de seguimiento-regulación, lo que sugiere que la información sobre las estrategias metacognitivas en la enseñanzaaprendizaje de las matemáticas es más rica y exhaustiva-, y la idoneidad de ciertos items.

Calderón y Chiecher (2012) trabajaron con un grupo de estudiantes de secundaria y con otro de estudiantes universitarios. Los autores reportaron una progresiva complejización en el uso de ciertas EA para el proceso de adquisición, recuperación y uso de procedimientos estratégicos para el aprendizaje a medida que los estudiantes avanzaban en los años de escolaridad.

En otro estudio se halló que entre el comienzo de la carrera y el quinto año los alumnos universitarios van cambiando sus métodos de estudio y estilos de aprendizaje. Comienzan memorizando los contenidos pero en el último año el aprendizaje parece volverse más reflexivo (Lockett, Ojeda y Gili, 2008).

Chiu, Wing-Yin y McBride-Chang (2007) analizaron datos del informe PISA 2000 en estudiantes de 15 años de varios paises. Estos investigadores reportan que el empleo de las EA de repetición y elaboración no muestra relación con el rendimiento, mientras que las estrategias metacognitivas correlacionan positivamente con el rendimiento en matemáticas. 


\section{Concepto y clasificación de las estrategias de aprendizaje}

En la literatura enfocada en las EA puede observarse un amplio abanico de definiciones y tipologías, por lo que se hace necesario situar el presente estudio dentro de una perspectiva teórica. Así, se decidió adoptar el concepto de EA propuesto por Wild (2000) en el cual las EA se relacionan con el control indirecto del aprendizaje a través de la influencia intencional de estados motivacionales y afectivos personales. Wild refiere que las EA son las formas en que la información se selecciona, adquiere, organiza e integra al conocimiento existente. Se destaca que el enfoque principal de la investigación de las EA hasta ahora es predominantemente en el dominio cognitivo.

Wild (2000) considera tres grandes grupos de estrategias que incluyen distintos procedimientos:

\section{Estrategias cognitivas}

El subconjunto de estas estrategias incluye procesos que sirven para adquirir, procesar y almacenar información directamente. Están incluidas las siguientes estrategias: repetitivas, organizacionales, de elaboración y de pensamiento crítico o de prueba.

Las estrategias de repetición o de memorización son aquellas actividades de aprendizaje que intentan lograr un anclaje sólido a largo plazo mediante la repetición activa de hechos individuales. Son, esencialmente, actividades de estudio que se enfocan en recordar hechos y reglas a través de una simple memorización.

Las estrategias organizacionales son actividades de aprendizaje que se ejecutan con el fin de reorganizar un material para que se aborde adecuadamente en el contexto de los objetivos de aprendizaje y el conocimiento previo. El objetivo de estas EA es transformar la información actual en una forma cognitivamente más fácil de procesar. Las estrategias organizacionales típicas incluyen la identificación de hechos y líneas de argumento importantes en un texto o la producción independiente de diagramas y bocetos para su ilustración. Las estrategias organizacionales conducen a una comprensión más profunda del tema.

Las estrategias de elaboración incluyen tareas de aprendizaje que son adecuadas para integrar activamente el conocimiento recién adquirido en la estructura de conocimiento existente. Es decir, sirven para incorporar los nuevos contenidos en una red de otras reservas de conocimiento de la memoria a largo plazo. Esto incluye, por ejemplo, el enriquecimiento verbal o pictórico del nuevo material y la vinculación del material recién aprendido con ejemplos cotidianos o experiencias personales.

Un segundo componente distinguible de las estrategias de elaboración se denomina prueba o pensamiento crítico. Estas son actividades de estudio que profundizan la comprensión del material a través de un cuestionamiento crítico de declaraciones y contextos de justificación. 


\section{Estrategias metacognitivas}

Mientras que las EA cognitivas se relacionan principalmente con los procesos de información inmediata al tratar con los contenidos de aprendizaje, las EA metacognitivas se centran en el autocontrol activo y consciente del propio aprendizaje. Se pueden distinguir tres subestrategias, que idealmente forman un bucle de control bien ajustado.

El primer paso de este ciclo de control consiste en la planificación activa de los propios pasos de aprendizaje. Dependiendo de la situación de aprendizaje, esto incluye una determinación más precisa de los contenidos que se van a aprender, una estimación de las secuencias de trabajo y una planificación consciente de los requisitos de tiempo.

El segundo paso se relaciona con el monitoreo activo del propio proceso de aprendizaje sobre la base de una comparación objetiva y real de las metas establecidas y del progreso alcanzado en el aprendizaje. Los estudiantes con un autocontrol intensivo de su éxito de aprendizaje, por ejemplo, se hacen preguntas sobre el material para asegurarse de que han comprendido todo. Asimismo, pueden explicar ciertas partes del material a sus compañeros de estudio para evaluar su propia comprensión.

El tercer paso completa sistemáticamente el ciclo de control. Este componente denominado regular los pasos de aprendizaje se refiere a la consideración deliberada de las dificultades de aprendizaje autodiagnóstico. Por ejemplo, en un nuevo intento, los estudiantes podrian trabajar a través de su contenido de aprendizaje, aparentemente poco aprendido, algo más lentamente, utilizando estrategias de aprendizaje cognitivo más sofisticadas tales como las estrategias de organización y/o elaboración.

\section{Estrategias relacionadas con los recursos}

Estas se refieren a las competencias de autogestión de los estudiantes que organizan actividades de aprendizaje en su conjunto. Las actividades incluidas aquí a menudo se encuentran en un lugar destacado en la literatura de asesoramiento relevante. Algunos autores también se refieren a ellas como estrategias de apoyo planteando una subdivisión en recursos externos e internos (Wild y Schiefele, 1994). La provisión de recursos internos se refiere a la gestión del propio esfuerzo: la administración de su propio presupuesto de tiempo así como el aumento de la atención y la concentración. En contraste, el uso de recursos externos consiste en el uso de fuentes de información como medios impresos o fuentes de información electrónica, y el uso activo del potencial del aprendizaje cooperativo. Otro aspecto es el diseño amigable de un entorno propicio para el aprendizaje. La Figura 1 muestra la descripción general de las estrategias de aprendizaje. 


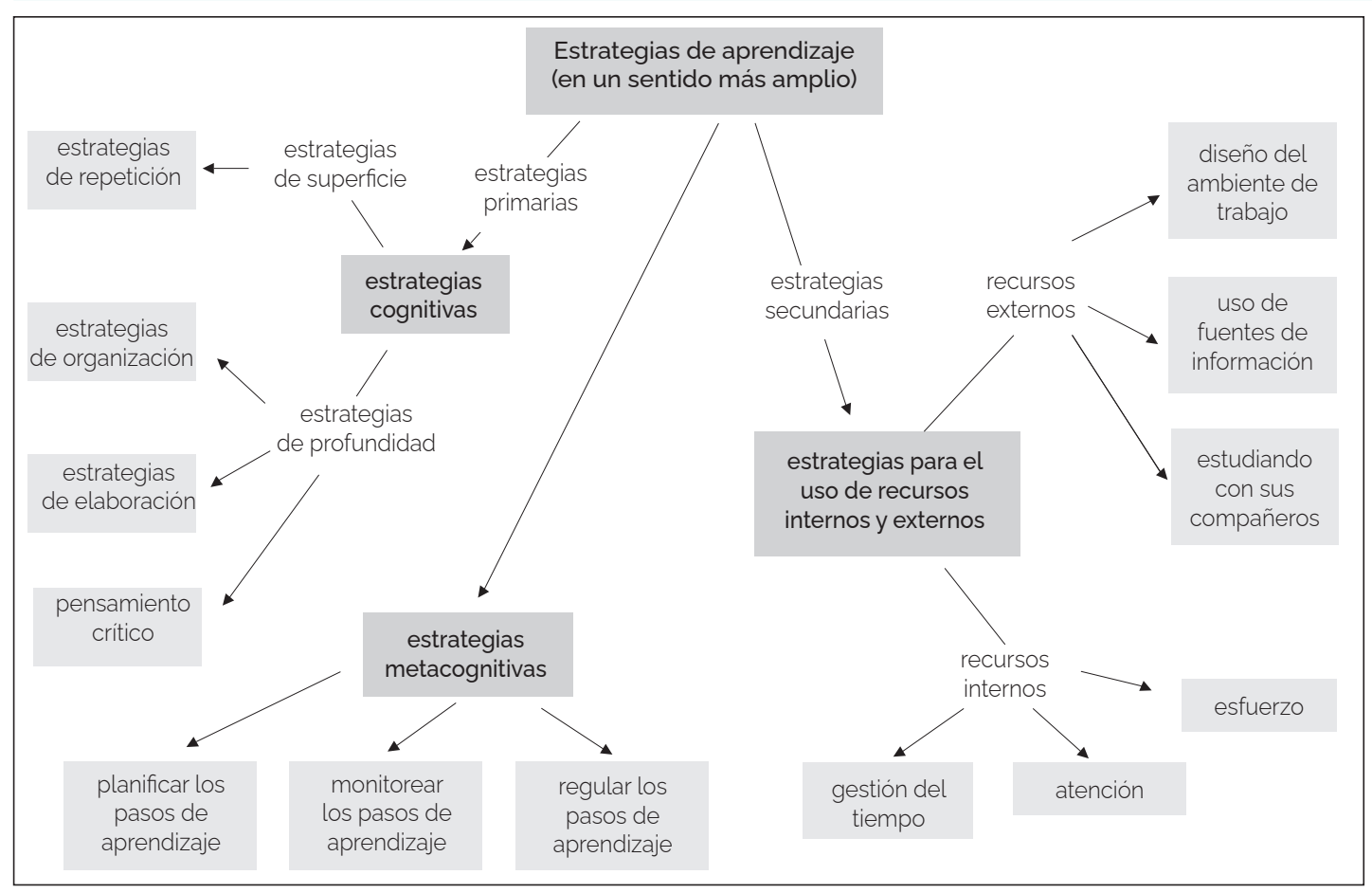

Fuente: Wild, 2005, p. 194, traducción de los autores.

El presente estudio se realizó con estudiantes de educación media superior denominada genéricamente como nivel bachillerato. Se juzga necesario mencionar algunas de sus características en México. La Educación Media Superior se compone de tres años y se ubica entre el nivel básico obligatorio, que actualmente tiene diez años, y la Educación Superior (cf. Villa, 2007). Se cursa después de los tres años del tercer nivel de educación básica y está organizada en dos modalidades: Bachillerato y Educación Profesional Técnica. Centrándonos en el primero diremos que a su vez se divide en General o Propedéutico y en Tecnológico o Bivalente. Entre ambos existen diferencias: el Bachillerato General se imparte en 13 tipos diferentes de instituciones (colegio de bachilleres, centros de estudios de bachillerato, Escuela Nacional Preparatoria, preparatorias oficiales y anexas a escuelas normales, entre otras) y su orientación consiste tanto en preparar a los estudiantes para continuar estudios superiores como en aportar el primer acercamiento del estudiante a las diferentes áreas académicas (ciencias sociales, naturales, matemáticas, etc.). El Bachillerato Tecnológico o Bivalente pretende capacitar al estudiante para continuar estudios superiores y para el desempeño de alguna actividad productiva. Se imparte en 12 tipos de instituciones diferentes.

Según Villa (2007), aunque la matrícula estudiantil en este nivel crece continuamente ya que absorbe a casi el 98\% de los egresados del nivel escolar previo - lo cual sugiere que el acceso ha mejorado en los últimos años-, se observan deficiencias importantes y se lo considera el eslabón más débil de la cadena educativa, destacando como principal problema la falta de permanencia estudiantil. No obstante, se han implementado dos procesos impulsados por el gobierno con el objetivo de mejorar las condiciones de acceso y la calidad del servicio educativo. Dichos procesos son la Reforma Integral de la Educación Media Superior (RIEMS) iniciada en el año 2007, y la garantía constitucional de brindar de manera obligatoria esta educación a la población demandante, establecida por decreto en 2012 (Rodriguez, 2018). Destacamos que del proyecto de reforma se han mantenido al margen los bachilleratos que ofrecen la Universidad Nacional Autónoma de México y el Instituto Politécnico Nacional. 


\section{Método}

Este estudio propone los siguientes objetivos:

1) Caracterizar el empleo de las EA en matemáticas por parte de alumnos mexicanos de bachillerato y;

2) Analizar posibles diferencias en función del curso académico y del sexo.

\section{Muestra}

La muestra de estudio estuvo constituida por varios grupos escolares escogidos del único plantel de bachillerato general ubicado en la ciudad de Apizaco perteneciente al Estado de Tlaxcala-México. Específicamente participaron alumnos del Plantel 10 del Colegio de Bachilleres del Estado de Tlaxcala (COBAT). Cabe decir que existe un total de 25 COBAT distribuidos en distintas ciudades de esta entidad federativa. Se escogió el plantel 10 por su disponibilidad geográfica para los autores de esta investigación. Al momento de iniciar el estudio en este colegio se hallaban inscritos 1216 alumnos. Los participantes se escogieron mediante un muestreo no probabilístico por conglomerados, que consistian en los grupos escolares. No se aplicó un muestreo probabilístico porque se buscó asegurar similitud entre los conglomerados elegidos de los distintos niveles escolares con respecto a la proporción de estudiantes masculinos y femeninos. De este modo participaron 154 estudiantes: 91 mujeres (59,1\%) y 63 varones $(40,9 \%)$, con una edad promedio de 16,4 años. En la Tabla 1 aparece la distribución detallada de la muestra en función del semestre, nivel escolar o curso, el grupo y el sexo de los participantes.

Tabla 1. Distribución de los participantes por semestre, grupo y sexo

\begin{tabular}{|c|c|c|c|c|}
\hline \multirow{2}{*}{ Semestre } & Grupo & Mujer & Varón & \multirow{2}{*}{ Total } \\
\cline { 3 - 4 } & 203 & 23 & 14 & 37 \\
\hline Segundo & 402 & 29 & 16 & 45 \\
\hline Cuarto & 413 & 28 & 19 & 47 \\
\hline Cuarto & 601 & 11 & 14 & 25 \\
Sexto & & 91 & 63 & 154 \\
\hline \multicolumn{2}{|c|}{ Total } & & &
\end{tabular}

Fuente: Elaboración propia.

Nota: El primer dígito del número del grupo indica el semestre que corresponde al grupo.

\section{Instrumento de medida}

Se empleó el cuestionario LIST (Estrategias de Aprendizaje en la Universidad) de Wild y Schiefele (1994), diseñado originalmente en alemán. LIST se centra en la evaluación de estrategias cognitivas, metacognitivas y estrategias relacionadas con los recursos mediante 13 dimensiones de estrategias de aprendizaje. LIST se ha modificado y probado varias veces. Abarca items generales que pueden aplicarse a todo tipo de temas.

Uno de los antecedentes del cuestionario LIST es el Cuestionario de Motivación y Estrategias de Aprendizaje (MSLQ) antes citado de Pintrich et al. (1993). La principal diferencia entre ambos es que el MSLQ pone más énfasis en incluir diferentes aspectos de la motivación como orientación a objetivos o control de creencias de aprendizaje. 
Un trabajo que influyó en una versión más reciente de LIST fue el instrumento Inventario de Estrategias de Aprendizaje y Estudio (Learning and Study Strategies Inventory - LASSI) de Weinstein y Palmer (2002) que también separa los aspectos cognitivos. Las escalas LASSI incluyen en parte los mismos contenidos que LIST aunque con nombres diferentes.

Wild y Schiefele (1994) y Griese et al. (2015) modificaron el cuestionario original LIST a 69 items y eliminaron la subescala denominada Chequeos Críticos (Critical Checks) porque no parecía apropiada para las matemáticas al comienzo de la universidad. Así, en esta investigación se utilizó el LIST de 12 dimensiones con 69 items dado que se ajustaba mejor a las caracteristicas de la muestra de participantes del estudio (Tabla 2).

Tabla 2. Dimensiones del cuestionario LIST y ejemplo de items

\begin{tabular}{|c|c|c|}
\hline Dimensión & $\begin{array}{l}\text { Número de } \\
\text { items }\end{array}$ & Ejemplo \\
\hline Organización & 8 & $\begin{array}{l}\text { Trato de ordenar el tema de tal manera } \\
\text { que se me facilite recordarlo. }\end{array}$ \\
\hline Elaboración & 8 & $\begin{array}{l}\text { Relaciono lo que estoy aprendiendo } \\
\text { con mis propias experiencias. }\end{array}$ \\
\hline Repetición & 7 & Leo mis apuntes varias veces seguidas. \\
\hline $\begin{array}{l}\text { Metacognición } \\
\text { Planificación }\end{array}$ & 4 & $\begin{array}{l}\text { Decido de antemano qué tanto de la } \\
\text { materia me gustaría trabajar en esta } \\
\text { sesión. }\end{array}$ \\
\hline Metacognición Control & 4 & $\begin{array}{l}\text { Trabajo en tareas extra con el fin de } \\
\text { cerciorarme de si realmente he com- } \\
\text { prendido el tema. }\end{array}$ \\
\hline $\begin{array}{l}\text { Metacognición } \\
\text { Regulación }\end{array}$ & 3 & $\begin{array}{l}\text { Cuando un aspecto parece confuso o } \\
\text { no claro lo examino de nuevo a fondo. }\end{array}$ \\
\hline Esfuerzo & 8 & $\begin{array}{l}\text { Hago un esfuerzo aunque el tema } \\
\text { puede no ser adecuado para mí. }\end{array}$ \\
\hline Atención & 6 & $\begin{array}{l}\text { Me encuentro pensando en cosas } \\
\text { completamente distintas. }\end{array}$ \\
\hline Gestión del tiempo & 4 & $\begin{array}{l}\text { Antes de cada período de estudio } \\
\text { designo la duración de mi trabajo. }\end{array}$ \\
\hline $\begin{array}{l}\text { Ambiente de } \\
\text { aprendizaje }\end{array}$ & 6 & $\begin{array}{l}\text { Cuando aprendo algo siempre me } \\
\text { siento en el mismo lugar. }\end{array}$ \\
\hline Aprendizaje en pares & 7 & $\begin{array}{l}\text { Recurro a la ayuda de otros cuando } \\
\text { tengo serios problemas para } \\
\text { comprender algo. }\end{array}$ \\
\hline $\begin{array}{l}\text { Uso de obras de } \\
\text { referencia }\end{array}$ & 4 & $\begin{array}{l}\text { Siempre que no entiendo un término } \\
\text { técnico lo busco en un libro de texto o } \\
\text { en Internet. }\end{array}$ \\
\hline
\end{tabular}

Fuente: Elaboración propia.

En 2011 Gómez-Chacón realizó una traducciónyadaptaciónalespañolde España de 34 items correspondientes a 6 dimensiones, organización, elaboración, repetición, metacognición de planificación, metacognición de control y metacognición de regulación, del cuestionario LIST. Esta se ha utilizado en distintas investigaciones en España y en estudios comparativos (p. ej. Gómez-Chacón et al., 2015) dentro 
del proyecto Investigación en Educación Matemática Universitaria (INVEDUMAT) del Instituto de Matemática Interdisciplinar (España) (IMI-2014-Actions Research Committee Grant UCM).

La traducción y adaptación al español mexicano de los restantes 35 ítems del cuestionario LIST de las otras 6 dimensiones (esfuerzo, atención, gestión del tiempo, aprendizaje en pares, ambiente de aprendizaje y uso de obras de referencia) fueron realizadas por los autores del presente trabajo. Para ello se tomaron en consideración las sugerencias de Gorard (2003) respecto al proceso de traducción-retraducción de un instrumento. De esta manera se recurrió a un especialista en traducción de textos en lengua inglesa.

La versión en español de los 69 ítems del LIST se sometió a una validez de contenido siguiendo los lineamientos de Escobar-Pérez y Cuervo-Martínez (2008), que contó con la participación de 4 jueces, 2 de ellos con experiencia en este tipo de procesos. Se destaca que los 4 son profesores en un posgrado en educación matemática, campo en donde se desarrollan trabajos con esta temática, entre otras.

Aunque un estudio en España mostró que el LIST mantiene sus cualidades cuando se utiliza en otro país (Gómez-Chacón et al., 2015), en nuestro estudio se realizó una aplicación piloto a 173 estudiantes de los últimos semestres de bachillerato distintos a los participantes de este estudio. El análisis de los datos mostró que el instrumento posee confiabilidad $(\alpha=0,93)$ y validez $(57,16 \%$ de varianza total explicada, con 12 factores).

Los ítems del LIST se responden en una escala tipo Likert de cinco puntos: $1=$ totalmente en desacuerdo, $2=e n$ desacuerdo, 3=indiferente, $4=$ de acuerdo y $5=$ totalmente de acuerdo. Cabe mencionarque en las instrucciones proporcionadas a los participantes se enfatizó que la serie de ítems del LIST describen situaciones relacionadas con la clase de matemáticas.

\section{Procedimiento}

Antes de realizar la recogida de datos se solicitó la autorización correspondiente ante la dirección del plantel educativo de los participantes. Posteriormente se les expuso tanto la naturaleza de la investigación como el hecho de que los datos obtenidos se tratarian de manera anónima. Los datos personales que se requirieron fueron la fecha de nacimiento y el sexo. Aunque dependió de cada estudiante, la realización de la prueba tuvo una duración de entre 15 y 20 minutos. La aplicación se realizó en forma grupal.

\section{Análisis de datos}

En primer lugar se volvió a evaluar la consistencia interna de las dimensiones mediante el alfa de Cronbach. Asimismo se analizaron los datos para detectar posibles diferencias en el empleo de estrategias de aprendizaje en matemáticas en función del semestre académico y del sexo.

Para el primer análisis se utilizó la prueba no paramétrica para k muestras independientes de Kruskal-Wallis y para un posible análisis post-hoc se consideró la prueba no paramétrica $U$ de Mann-Whitney. Estas pruebas estadisticas se eligieron porque los datos recopilados no cumplían con los criterios de normalidad y homocedasticidad. Para estimar la magnitud de las diferencias se calculó el tamaño del efecto, denotado por el parámetro r. El coeficiente obtenido se 
interpretó con base en el siguiente criterio: $r=0,10$, tamaño del efecto débil, $r=0,30$, tamaño del efecto moderado y a partir de $r=0,50$, tamaño del efecto fuerte (Field, 2013). El análisis estadistico se realizó con el software IBM SPSS Statistics 20.

\section{Resultados}

Primero, el coeficiente de confiabilidad obtenido se interpretó con el criterio propuesto por Ruiz (2015). El coeficiente obtenido sugiere una confiabilidad muy alta (Tabla 3).

Tabla 3. Coeficiente de Alfa de Cronbach.

\begin{tabular}{|c|c|}
\hline $\begin{array}{c}\text { Alfa de } \\
\text { Cronbach }\end{array}$ & $\begin{array}{c}\text { N de } \\
\text { elementos }\end{array}$ \\
\hline 0,94 & 154 \\
\hline
\end{tabular}

Fuente: Elaboración propia.

El alfa de Cronbach ( $\alpha$ ) se utilizó para evaluar las 12 dimensiones del LIST. Los resultados obtenidos se muestran en la Tabla 4. La consistencia interna de las escalas resultó aceptable en cinco dimensiones que presentan confiabilidades iguales o superiores a 0,70 .

Tabla 4. Dimensiones de las estrategias de aprendizaje del cuestionario LIST

\begin{tabular}{cccccc}
\hline Dimensión & $\begin{array}{c}\text { Número } \\
\text { de items }\end{array}$ & $\begin{array}{c}\text { Alfa de } \\
\text { Cronbach } \\
\text { Original }\end{array}$ & $\begin{array}{c}\text { Alfa de } \\
\text { Cronbach } \\
\text { Investigación }\end{array}$ & Media & $\begin{array}{c}\text { Desviación } \\
\text { Tipica }\end{array}$ \\
\hline Organización & 8 & 0,82 & 0,83 & 3,57 & 0,70 \\
Elaboración & 8 & 0,77 & 0,76 & 3,56 & 0,60 \\
$\begin{array}{c}\text { Repetición } \\
\text { Met. Planificación }\end{array}$ & 7 & 0,73 & 0,70 & 3,34 & 0,61 \\
Met. Control & 4 & 0,64 & 0,51 & 3,45 & 0,66 \\
$\begin{array}{c}\text { Met. Regulación } \\
\text { Esfuerzo }\end{array}$ & 3 & 0,64 & 0,59 & 3,10 & 0,74 \\
Atención & 8 & 0,64 & 0,59 & 3,72 & 0,58 \\
$\begin{array}{c}\text { Gestión del } \\
\text { tiempo }\end{array}$ & 4 & 0,90 & 0,67 & 3,46 & 0,58 \\
$\begin{array}{c}\text { Ambiente de } \\
\text { aprendizaje }\end{array}$ & 6 & 0,83 & 0,85 & 3,18 & 0,90 \\
$\begin{array}{c}\text { Aprendizaje en } \\
\text { pares }\end{array}$ & 7 & 0,71 & 0,63 & 3,24 & 0,77 \\
$\begin{array}{c}\text { Uso de obras de } \\
\text { referencia }\end{array}$ & 4 & 0,82 & 0,66 & 3,65 & 0,64 \\
\hline
\end{tabular}

Fuente: Elaboración propia. 


\section{Diferencias en función del curso académico: segundo, cuarto y sexto semestre}

Las diferencias en función del curso académico se analizaron mediante la prueba no paramétrica de Kruskal-Wallis comparando las medias en cada estrategia por semestre. Los datos se muestran en la Tabla 5.

Tabla 5. Análisis de las diferencias en función del curso académico

\begin{tabular}{|c|c|c|c|c|c|c|}
\hline \multirow[b]{2}{*}{ Estrategia } & \multirow[b]{2}{*}{ Grupo } & \multirow[b]{2}{*}{ Media } & \multirow[b]{2}{*}{ Desv. T. } & \multicolumn{3}{|c|}{ Prueba de Kruskal-Wallis } \\
\hline & & & & $X^{2}$ & gl & $\mathrm{p}$ \\
\hline \multirow{4}{*}{ Organización } & 203 & 3.72 & 0,86 & \multirow{4}{*}{20,19} & \multirow{4}{*}{3} & \multirow{4}{*}{0,000} \\
\hline & 402 & 3.78 & 0,47 & & & \\
\hline & 413 & 3,10 & 0,66 & & & \\
\hline & 601 & 3,51 & 0,65 & & & \\
\hline \multirow{4}{*}{ Elaboración } & 203 & 3.70 & 0,67 & \multirow{4}{*}{10,03} & \multirow{4}{*}{3} & \multirow{4}{*}{0,018} \\
\hline & 402 & 3,71 & 0,53 & & & \\
\hline & 413 & 3,36 & 0,51 & & & \\
\hline & 601 & 3,42 & 0,59 & & & \\
\hline \multirow{4}{*}{ Repetición } & 203 & 3.54 & 0,71 & \multirow{4}{*}{18,91} & \multirow{4}{*}{3} & \multirow{4}{*}{0,000} \\
\hline & 402 & 3,41 & 0,50 & & & \\
\hline & 413 & 2,90 & 0,50 & & & \\
\hline & 601 & 3.34 & 0,59 & & & \\
\hline \multirow{4}{*}{ Met. Planificación } & 203 & 3.56 & 0,74 & \multirow{4}{*}{5,04} & \multirow{4}{*}{3} & \multirow{4}{*}{0,169} \\
\hline & 402 & 3.52 & 0,63 & & & \\
\hline & 413 & 3,27 & 0,53 & & & \\
\hline & 601 & 3.38 & 0,68 & & & \\
\hline \multirow{4}{*}{ Met. Control } & 203 & 3,27 & 0,87 & \multirow{4}{*}{17,09} & \multirow{4}{*}{3} & \multirow{4}{*}{0,001} \\
\hline & 402 & 3.31 & 0,49 & & & \\
\hline & 413 & 2,70 & 0,66 & & & \\
\hline & 601 & 2,97 & 0,64 & & & \\
\hline \multirow{4}{*}{ Met. Regulación } & 203 & 3.93 & 0,84 & \multirow{4}{*}{8,60} & \multirow{4}{*}{3} & \multirow{4}{*}{0,035} \\
\hline & 402 & 3,80 & 0,57 & & & \\
\hline & 413 & 3,45 & 0,73 & & & \\
\hline & 601 & 3,62 & 0,78 & & & \\
\hline \multirow{4}{*}{ Esfuerzo } & 203 & 3,46 & 0,67 & \multirow{4}{*}{2,14} & & \\
\hline & 402 & 3.54 & 0,43 & & 3 & 0,544 \\
\hline & 413 & 3,32 & 0,62 & & & \\
\hline & 601 & 3,45 & 0,63 & & & \\
\hline
\end{tabular}




\begin{tabular}{|c|c|c|c|c|c|c|}
\hline \multirow{4}{*}{ Atención } & 203 & 3,27 & 0,92 & \multirow{4}{*}{0,96} & \multirow{4}{*}{3} & \multirow{4}{*}{0,810} \\
\hline & 402 & 3,16 & 0,94 & & & \\
\hline & 413 & 3,04 & 1,00 & & & \\
\hline & 601 & 3,21 & 0,82 & & & \\
\hline \multirow{4}{*}{$\begin{array}{l}\text { Gestión del } \\
\text { tiempo }\end{array}$} & 203 & 3,29 & 0,72 & \multirow{4}{*}{5,09} & \multirow{4}{*}{3} & \multirow{4}{*}{0,165} \\
\hline & 402 & 3,42 & 0,73 & & & \\
\hline & 413 & 3,00 & 0,85 & & & \\
\hline & 601 & 3,16 & 0,77 & & & \\
\hline \multirow{4}{*}{$\begin{array}{l}\text { Ambiente de } \\
\text { aprendizaje }\end{array}$} & 203 & 3,61 & 0,70 & \multirow{4}{*}{3,95} & \multirow{4}{*}{3} & \multirow{4}{*}{0,267} \\
\hline & 402 & 3.70 & 0,60 & & & \\
\hline & 413 & 3,43 & 0,64 & & & \\
\hline & 601 & 3.74 & 0,61 & & & \\
\hline \multirow{4}{*}{$\begin{array}{l}\text { Aprendizaje en } \\
\text { pares }\end{array}$} & 203 & 3,61 & 0,72 & \multirow{4}{*}{9,30} & \multirow{4}{*}{3} & \multirow{4}{*}{0,025} \\
\hline & 402 & 3,43 & 0,63 & & & \\
\hline & 413 & 3,21 & 0,67 & & & \\
\hline & 601 & 3,62 & 0,50 & & & \\
\hline \multirow{4}{*}{$\begin{array}{l}\text { Uso de obras de } \\
\text { referencia }\end{array}$} & 203 & 3,89 & 0,91 & \multirow{4}{*}{6,78} & \multirow{4}{*}{3} & \multirow{4}{*}{0,079} \\
\hline & 402 & 3,83 & 0,59 & & & \\
\hline & 413 & 3,36 & 0,89 & & & \\
\hline & 601 & 3.78 & 0,77 & & & \\
\hline
\end{tabular}

Fuente: Elaboración propia.

Se encontró que las diferencias son estadisticamente significativas en las estrategias de organización, elaboración, repetición, metacognitiva de control, metacognitiva de regulación y aprendizaje en pares.

Con el propósito de profundizar en los resultados obtenidos donde hubo diferencias significativas se procedió a realizar un análisis post-hoc con la prueba $U$ de Mann-Whitney. En la Tabla 6 se presentan los resultados al analizar al grupo de segundo semestre (203) y a uno de cuarto semestre (402).

Tabla 6. Diferencias post-hoc entre los grupos 203 y 402

\begin{tabular}{|c|c|c|c|c|}
\hline & \multicolumn{4}{|c|}{ Test U de Mann-Whitney } \\
\hline Estrategia & $U$ & $Z$ & $\mathrm{P}$ & $\mathrm{r}$ \\
\hline Organización & 735,00 & $-0,911$ & 0,362 & $-0,10$ \\
& & 20.19 & 3 & .000 \\
\hline Elaboración & 774,00 & $-0,547$ & 0,585 & $-0,06$ \\
\hline Repetición & 666,00 & $-1,559$ & 0,119 & $-0,17$ \\
\hline Met. Control & 784,00 & $-0,456$ & 0,649 & $-0,05$ \\
\hline Met. Regulación & 661,50 & $-1,164$ & 0,106 & $-0,12$ \\
\hline Aprendizaje en pares & 649,50 & $-1,711$ & 0,087 & $-0,18$ \\
\hline
\end{tabular}

Fuente: Elaboración propia. 
No se observaron diferencias estadisticamente significativas en los puntajes obtenidos en las estrategias evaluadas. Los hallazgos indican un empleo similar en estas EA.

No obstante los resultados del análisis anterior donde se contrastó el grupo de segundo semestre con uno de cuarto semestre, al analizar los datos de los alumnos de ese grupo de segundo semestre con el grupo 413 de cuarto semestre se hallaron diferencias estadisticamente significativas en las seis estrategias evaluadas. Los resultados aportan evidencia de que los estudiantes de segundo semestre muestran tendencia a recurrir más al uso estas estrategias que los de cuarto semestre (Tabla 7).

Tabla 7. Diferencias post-hoc entre los grupos 203 y 413

\begin{tabular}{|c|c|c|c|c|}
\hline & \multicolumn{5}{|c|}{ Test U de Mann-Whitney } \\
\hline Estrategia & $U$ & Z & $\mathrm{p}$ & $\mathrm{r}$ \\
\hline Organización & 222,00 & $-3,456$ & 0,001 & $-0,43$ \\
& & & & .000 \\
\hline Elaboración & 293,50 & $-2,434$ & 0,015 & $-0,30$ \\
\hline Repetición & 199,00 & $-3,799$ & 0,000 & $-0,48$ \\
\hline Met. Control & 262,00 & $-2,894$ & 0,004 & $-0,36$ \\
\hline Met. Regulación & 275,50 & $-2,711$ & 0,007 & $-0,34$ \\
\hline Aprendizaje en pares & 307,00 & $-2,237$ & 0,025 & $-0,28$ \\
\hline
\end{tabular}

Fuente: Elaboración propia.

Al analizar las diferencias observadas en el uso de las EA entre los participantes de segundo semestre con los de sexto semestre, es decir, el grupo 601, se encontró que únicamente existen diferencias estadísticamente significativas en las estrategias de organización, de elaboración y metacognitiva de control, véase la Tabla 8. Nuevamente, al igual que en los análisis anteriores, los resultados sugieren que los alumnos de segundo semestre hacen más uso de estas estrategias.

Tabla 8. Diferencias post-hoc entre los grupos 203 y 601

\begin{tabular}{|c|c|c|c|c|}
\hline & \multicolumn{5}{|c|}{ Test U de Mann-Whitney } \\
\hline Estrategia & $U$ & Z & $\mathrm{P}$ & $\mathrm{r}$ \\
\hline Organización & 640,50 & $-2,069$ & 0,039 & $-0,22$ \\
& & & & .000 \\
\hline Elaboración & 616,00 & $-2,293$ & 0,022 & $-0,25$ \\
\hline Repetición & 704,00 & $-1,499$ & 0,134 & $-0,16$ \\
\hline Met. Control & 637,50 & $-2,103$ & 0,035 & $-0,23$ \\
\hline Met. Regulación & 657,00 & $-1,938$ & 0,053 & $-0,21$ \\
\hline Aprendizaje en pares & 827,50 & $-0,380$ & 0,704 & $-0,04$ \\
\hline
\end{tabular}

Fuente: Elaboración propia.

También se contrastaron los datos obtenidos en los dos grupos de alumnos de cuarto semestre (Tabla 9). 


\begin{tabular}{|c|c|c|c|c|}
\hline & \multicolumn{4}{|c|}{ Test U de Mann-Whitney } \\
\hline Estrategia & $U$ & $Z$ & $p$ & $r$ \\
\hline Organización & 223,00 & $-4,175$ & 0,000 & $-0,49$ \\
& & & & .000 \\
\hline Elaboración & 373,00 & $-2,330$ & 0,020 & $-0,27$ \\
\hline Repetición & 269,50 & $-3,606$ & 0,000 & $-0,43$ \\
\hline Met. Control & 262,50 & $-3,709$ & 0,000 & $-0,44$ \\
\hline Met. Regulación & 421,00 & $-1,758$ & 0,079 & $-0,21$ \\
\hline Aprendizaje en pares & 424,50 & $-1,698$ & 0,090 & $-0,20$ \\
\hline
\end{tabular}

Fuente: Elaboración propia.

Los resultados indican que hay diferencias estadisticamente significativas en todas las estrategias excepto en la metacognitiva de regulación y en la de aprendizaje en pares. En las estrategias de organización, elaboración, repetición y metacognitiva de control el grupo 402 obtuvo puntajes medios más altos que los alumnos del grupo 413.

Se examinaron asimismo las diferencias en el uso de las EA entre los participantes de los dos grupos de cuarto semestre y los de sexto semestre. En la Tabla 10 aparecen los resultados del análisis de los grupos 402 y 601.

Tabla 10. Diferencias post-hoc entre los grupos 402 y 601

\begin{tabular}{|c|c|c|c|c|}
\hline & \multicolumn{4}{|c|}{ Test U de Mann-Whitney } \\
\hline Estrategia & $U$ & Z & $\mathrm{p}$ & $\mathrm{r}$ \\
\hline Organización & 834,50 & $-1,750$ & 0,080 & $-0,18$ \\
& & & & .000 \\
\hline Elaboración & 836,50 & $-1,734$ & 0,083 & $-0,18$ \\
\hline Repetición & 1053,50 & $-0,031$ & 0,975 & $-0,00$ \\
\hline Met. Control & 724,00 & $-2,628$ & 0,009 & $-0,27$ \\
\hline Met. Regulación & 965,00 & $-0,730$ & 0,465 & $-0,07$ \\
\hline Aprendizaje en pares & 854,00 & $-1,596$ & 0,111 & $-0,16$ \\
\hline
\end{tabular}

Fuente: Elaboración propia.

Solamente se observan diferencias significativas en la estrategia metacognitiva de control. Los resultados sugieren que los alumnos de cuarto semestre hacen un mayor empleo de esta EA.

En la Tabla 11 se exponen los resultados del análisis de las diferencias realizadas entre los grupos 413 y 601 . 
Tabla 11. Diferencias post-hoc entre los grupos 413 y 601

\begin{tabular}{|c|c|c|c|c|}
\hline & \multicolumn{5}{|c|}{ Test U de Mann-Whitney } \\
\hline Estrategia & $U$ & Z & p & $r$ \\
\hline Organización & 372,50 & $-2,550$ & 0,011 & $-0,30$ \\
& & & & .000 \\
\hline Elaboración & 517,00 & $-0,838$ & 0,402 & $-0,09$ \\
\hline Repetición & 320,00 & $-3,175$ & 0,001 & $-0,37$ \\
\hline Met. Control & 465,00 & $-1,462$ & 0,144 & $-0,17$ \\
\hline Met. Regulación & 502,00 & $-1,021$ & 0,307 & $-0,12$ \\
\hline Aprendizaje en pares & 376,50 & $-2,504$ & 0,012 & $-0,29$ \\
\hline
\end{tabular}

Fuente: Elaboración propia.

Los resultados indican que hay diferencias estadisticamente significativas en todas las estrategias, excepto en las de elaboración, metacognitiva de control y metacognitiva de regulación. Siempre el grupo de más escolaridad obtiene medias aritméticas superiores al grupo de cuarto semestre lo cual significa que los de sexto utilizan más estas estrategias.

\section{Diferencias en función del sexo}

El estudio además pretendía explorar las diferencias en el uso de las EA entre estudiantes masculinos y femeninos. Con tal propósito se llevó a cabo un análisis con la prueba $U$ de Mann-Whitney, en la Tabla 12 se exhiben los resultados.

Tabla 12. Diferencias en función del sexo.

\begin{tabular}{|c|c|c|c|c|c|c|c|}
\hline \multirow[b]{2}{*}{ Estrategia } & \multirow[b]{2}{*}{ Sexo } & \multirow[b]{2}{*}{ Media } & \multirow{2}{*}{$\begin{array}{c}\text { Desv. } \\
\text { Típ. }\end{array}$} & \multicolumn{4}{|c|}{ Test U de Mann-Whitney } \\
\hline & & & & U & Z & $\mathrm{p}$ & $r$ \\
\hline \multirow[t]{2}{*}{ Organización } & Femenino & 3,69 & 0,75 & \multirow[b]{2}{*}{2011,50} & \multirow[b]{2}{*}{$-3,150$} & \multirow[b]{2}{*}{0,002} & \multirow[b]{2}{*}{$-0,25$} \\
\hline & Masculino & 3,41 & 0,59 & & & & \\
\hline \multirow[t]{2}{*}{ Elaboración } & Femenino & 3.54 & 0,63 & \multirow[b]{2}{*}{2739,50} & \multirow[b]{2}{*}{$-0,468$} & \multirow[b]{2}{*}{0,640} & \multirow[b]{2}{*}{$-0,03$} \\
\hline & Masculino & 3,60 & 0,55 & & & & \\
\hline \multirow[t]{2}{*}{ Repetición } & Femenino & 3,39 & 0,63 & \multirow[b]{2}{*}{2493,50} & \multirow[b]{2}{*}{$-1,376$} & \multirow[b]{2}{*}{0,169} & \multirow[b]{2}{*}{$-0,11$} \\
\hline & Masculino & 3,26 & 0,59 & & & & \\
\hline \multirow{2}{*}{$\begin{array}{c}\text { Met. } \\
\text { Planificación }\end{array}$} & Femenino & 3,48 & 0,65 & \multirow[b]{2}{*}{2601,50} & \multirow[b]{2}{*}{$-0,983$} & \multirow[b]{2}{*}{0,326} & \multirow[b]{2}{*}{$-0,07$} \\
\hline & Masculino & 3.40 & 0,68 & & & & \\
\hline \multirow[t]{2}{*}{ Met. Control } & Femenino & 3,12 & 0,70 & \multirow[b]{2}{*}{2680,00} & \multirow[b]{2}{*}{$-0,690$} & \multirow[b]{2}{*}{0,490} & \multirow[b]{2}{*}{$-0,05$} \\
\hline & Masculino & 3,06 & 0,70 & & & & \\
\hline \multirow{2}{*}{$\begin{array}{l}\text { Met. } \\
\text { Regulación }\end{array}$} & Femenino & 3.75 & 0,78 & \multirow[b]{2}{*}{2674,00} & \multirow[b]{2}{*}{$-0,715$} & \multirow[b]{2}{*}{0,475} & \multirow[b]{2}{*}{$-0,05$} \\
\hline & Masculino & 3,68 & 0,70 & & & & \\
\hline
\end{tabular}




\begin{tabular}{|c|c|c|c|c|c|c|c|}
\hline \multirow[t]{2}{*}{ Esfuerzo } & Femenino & 3.50 & 0,60 & \multirow[b]{2}{*}{2600,50} & \multirow[b]{2}{*}{$-0,980$} & \multirow[b]{2}{*}{0,327} & \multirow[b]{2}{*}{$-0,07$} \\
\hline & Masculino & 3,40 & 0,56 & & & & \\
\hline \multirow[t]{2}{*}{ Atención } & Femenino & 3,25 & 0,88 & \multirow[b]{2}{*}{2503,00} & \multirow[b]{2}{*}{$-1,338$} & \multirow[b]{2}{*}{0,181} & \multirow[b]{2}{*}{$-0,10$} \\
\hline & Masculino & 3,09 & 0,94 & & & & \\
\hline \multirow{2}{*}{$\begin{array}{l}\text { Gestión del } \\
\text { tiempo }\end{array}$} & Femenino & 3.30 & 0,70 & \multirow[b]{2}{*}{2609,00} & \multirow[b]{2}{*}{$-0,952$} & \multirow[b]{2}{*}{0,341} & \multirow[b]{2}{*}{$-0,07$} \\
\hline & Masculino & 3,15 & 0,86 & & & & \\
\hline \multirow{2}{*}{$\begin{array}{l}\text { Ambiente de } \\
\text { aprendizaje }\end{array}$} & Femenino & 3.74 & 0,64 & \multirow[b]{2}{*}{2240,00} & \multirow[b]{2}{*}{$-2,310$} & \multirow[b]{2}{*}{0,021} & \multirow[b]{2}{*}{$-0,18$} \\
\hline & Masculino & 3.52 & 0,62 & & & & \\
\hline \multirow{2}{*}{$\begin{array}{c}\text { Aprendizaje en } \\
\text { pares }\end{array}$} & Femenino & 3,54 & 0,63 & \multirow[b]{2}{*}{2543,50} & \multirow[b]{2}{*}{$-1,190$} & \multirow[b]{2}{*}{0,234} & \multirow[b]{2}{*}{$-0,09$} \\
\hline & Masculino & 3,42 & 0,64 & & & & \\
\hline \multirow{2}{*}{$\begin{array}{l}\text { Uso de obras } \\
\text { de referencia }\end{array}$} & Femenino & 3.91 & 0,72 & \multirow[b]{2}{*}{2086,50} & \multirow[b]{2}{*}{$-2,883$} & \multirow[b]{2}{*}{0,004} & \multirow[b]{2}{*}{$-0,23$} \\
\hline & Masculino & 3.51 & 0,84 & & & & \\
\hline
\end{tabular}

Fuente: Elaboración propia.

De las 12 estrategias evaluadas únicamente se encontraron diferencias estadisticamente significativas en las estrategias de organización, ambiente de aprendizaje y uso de obras de referencia, destacando que el uso es predominante en las alumnas. En lo que respecta a la ausencia de diferencias en las otras estrategias se puede plantear que los resultados sugieren un empleo similar en las demás estrategias en ambos sexos.

\section{Discusión y conclusiones}

Se ha sugerido que las EA pueden constituir el motor del aprendizaje (GascoTxabarri, 2016). En el ámbito de la enseñanza y aprendizaje de las matemáticas el empleo de las EA tiene repercusión en el razonamiento, en la resolución de problemas verbales y su resolución entre otros diversos. Las EA fomentan la autonomía y pueden ayudar a tomar decisiones en esta tarea matemática, como señala Gasco-Txabarri (2017a).

Por ello es necesario y relevante identificar a las EA para saber cuáles están más desarrolladas y cuáles requieren atención para trabajar en fomentarlas.

Los estudios realizados por investigadores de otros países (Kecici y Aydin, 2019; Gasco-Txabarri, 2016; Gómez-Chacón, et al. 2015; Griese, et al. 2015) sugieren que las EA de los estudiantes en matemáticas son áreas importantes para el área de la educación matemática y necesitan atención dentro del contexto mexicano.

Hasta el momento, el presente estudio es el único sobre EA en matemáticas en estudiantes mexicanos de nivel medio superior. Consideramos que el hecho de que este tema haya sido escasamente explorado en México confiere relevancia a este trabajo.

Entre los hallazgos obtenidos está, en primer lugar, que el cuestionario LIST tiene una confiabilidad muy aceptable en todos sus items y una confiabilidad aceptable en cinco de las dimensiones que evalúa, que son: organización, elaboración, repetición, atención y uso de obras de referencia. Esto arroja una señal positiva sobre la potencial utilidad del instrumento para futuros estudios sobre EA en el nivel medio superior. 
Asimismo, se encontró que las mujeres participantes tienen tendencia a gestionar mejor los saberes matemáticos, a contar con un ambiente de aprendizaje adecuado y a la búsqueda de información en diferentes fuentes ante dificultades, en mayor medida que los varones. Estos resultados coinciden con la evidencia de Bidjerano (2005) y Gasco-Txabarri (2017b) donde se verifica una mayor planificación del aprendizaje y autocontrol por parte de las alumnas en educación secundaria. La EA de organización, referente a la manera de gestionar el aprendizaje, se presentó en este trabajo más en mujeres estudiantes, de igual manera que en el estudio de Gasco-Txabarri (2017b) en la escuela secundaria; es decir, hay evidencia de que esta estrategia es usada por las mujeres en diferentes niveles educativos. Por otro lado, en la etapa universitaria las mujeres solicitan más ayuda que los varones cuando tienen una dificultad en matemáticas (Virtanen y Nevgi, 2010). En lo que respecta a las diferencias en función del curso o semestre académico se hallaron algunas estadisticamente significativas en las EA de organización, elaboración, repetición, metacognitiva de control, metacognitiva de regulación y aprendizaje en pares.

El análisis de los datos de los grupos 203 y 402 mostró un empleo similar en las EA de organización, elaboración, repetición, metacognitiva de control, metacognitiva de regulación y aprendizaje en pares.

En el análisis de los grupos 203 y 413, el alumnado del segundo semestre muestra una tendencia mayor, en comparación al de cuarto semestre, a recurrir a las EA de organización, elaboración, repetición, metacognitiva de control, metacognitiva de regulación y aprendizaje en pares.

En lo que respecta a los grupos 203 y 601, el grupo 203, perteneciente al segundo semestre de bachillerato, tiende más que el de sexto a recurrir a las EA de organización, elaboración y metacognitiva de control.

En la comparación de los grupos 402 y 413, los alumnos del 402 de cuarto semestre muestran un uso mayor de las EA de organización, elaboración, repetición y metacognitiva de control en mayor medida que el 413.

Entre los grupos 402 y 601, el alumnado del 402 recurre en mayor medida a la EA metacognitiva de control en comparación con los estudiantes del 601.

Al contrastar los datos de los grupos 413 y 601 resultó que los participantes del 601 emplean más las EA de organización, repetición y aprendizaje en pares.

El alumnado de segundo semestre del grupo 203 tiene tendencia a gestionar u organizar mejor los saberes matemáticos, relaciona el saber matemático con otras materias, memoriza lo aprendido, conoce y regula eficazmente los procesos cognitivos y se da el aprendizaje en interacción con otros estudiantes en mayor medida que en los demás grupos.

La evidencia de que existe un mayor uso de estrategias de aprendizaje a medida que el curso escolar aumenta (Gasco-Txabarri, 2016) se observa en este estudio principalmente al analizar los datos de los grupos de cuarto y sexto semestre, es decir, los grupos 413 y 601, respectivamente.

Se insiste en que parte de los resultados encontrados permiten plantear que el cuestionario LIST representa un instrumento confiable para implementar futuras investigaciones en población estudiantil mexicana sobre EA en matemáticas en el nivel medio y superior. 
En este mismo sentido, en el futuro podría estudiarse la relación del uso de las EA medidas con el cuestionario LIST y el rendimiento académico en matemáticas, específicamente qué tipo de EA corresponde a un mayor rendimiento en matemáticas.

Nota:

Aprobación final del artículo: editora responsable Mag. Verónica Zorrilla de San Martín.

Contribución de autoria: los autores han colaborado en partes iguales en todas las etapas del articulo. 


\section{Bibliografía}

BELTRÁN, J. (2003). Estrategias de aprendizaje. Revista de Educación, 332, 5573. $\quad$ http://www.educacionyfp.gob.es/dam/jcr:obc115bf-2ee5-4894-91f57e32e07059d4/re3320411443-pdf.pdf

BELTRÁN, J. (1993). Procesos, estrategias y técnicas de aprendizaje. Madrid: Síntesis.

BIDJERANO, T. (2005). Gender differences in self-regulated learning [Diferencias de género en el aprendizaje autorregulado]. Artículo presentado en The Annual Meeting of the Northeastern Educational Research Association. 19-21 Octubre. Nueva York. https://files.eric.ed.gov/fulltext/ED490777.pdf

BOERNER, S., SEEBER, G., KELLER, H. \& BEINBORN, P. (2005). Lernstrategien und lernerfolg im stadium IEstrategias de aprendizaje y éxito en el aprendizaje en los estudios]. Zeitschrift für Entwicklungspsychologie und Pädagogische Psychologie, 37(1), 17-26. https://dx.doi.org/10.1026/0049-8637.37.1.17

CALDERÓN, L. Y CHIECHER, A. (2012). Estrategias de aprendizaje, ¿procesos en construcción? Comparando el desempeño estratégico en educación secundaria y universitaria. Revista Electrónica Actualidades Investigativas en Educación, 12(2), 1-16. https://revistas.ucr.ac.cr/index.php/aie/article/view/1027

CHIU, M. M., WING-YIN, B. Y MCBRIDE-CHANG, C. (2007). Universals and specifics in learning strategies: Explaining adolescent mathematics, science, and reading achievement across 34 countries IUniversales y especificos en las estrategias de aprendizaje: explicar los logros de los adolescentes en matemáticas, ciencias y lectura en 34 paises]. Learning and Individual Differences, 17, 344-365.

http://dx.doi.org/10.1016/j.lindif.2007.03.007

ESCOBAR-PÉREZ, J., y CUERVO-MARTínEZ, Á. (2008). Validez de contenido y juicio de expertos: una aproximación a su utilización. Avances en medición, 6(1), 27-36. http://www.humanas.unal.edu.co/psicometria/files/7113/8574/5708/ Articulo3_Juicio_de_expertos_27-36.pdf

FIELD, A. (2013). Discovering statistics using IBM SPSS statistics IDescubrimiento de estadisticasutilizandoestadisticas deIBMSPSSI.Sage.https://WWW.researchgate. net/profile/Abdelrahman_Zueter2/post/What_are_the_conditions_for_using_ Ordinal_Logistic_regression_Can_anyone_share_the_various_regression_ methods_and_their_application/attachment/59d637d8c49f478072ea5080/ AS\%3A273691429015552\%401442264529487/download/ DISCOVERING+STATISTICS.Pdf

GASCO-TXABARRI, J. (2016). El empleo de estrategias en el aprendizaje de las matemáticas en la enseñanza secundaria obligatoria. Revista de Investigación Educativa, 34(2), 487-502. http://dx.doi.org/10.6018/rie.34.2.222901

GASCO-TXABARRI, J., ROS, I. Y GOÑI, A. (2017). A questionnaire on mathematics learning strategies (CEAMA): measurement and properties of an adaptation into Spanish: measurement and properties of an adaptation into Spanish / Cuestionario de Estrategias de Aprendizaje para las Matemáticas (CEAMA): medida y propiedades de una adaptación en lengua castellana. Cultura y Educación, 29(1), 183-209. http://dx.doi.org/10.1080/11356405.2016.1274145 
GASCO-TXABARRI, J. (2017a). La resolución de problemas aritmético-algebraicos y las estrategias de aprendizaje en matemáticas. Un estudio en educación secundaria obligatoria (ESO). Revista latinoamericana de investigación en matemática educativa, 20(2), 167-192. http://www.scielo.org.mx/pdf/relime/ v20n2/2007-6819-relime-20-02-167.pdf

GASCO-TXABARRI, J. (2017b). Diferencias en el uso de estrategias en el aprendizaje de las matemáticas en enseñanza secundaria según el sexo. Cuadernos de Investigación Educativa, 8(1), 47-59. http://dx.doi.org/10.18861/cied.2017.8.1.2638

GÓMEZ-CHACÓN, I. M., GRIESE, B., RÖSKEN-WINTER, B. Y GONZÁLEZ-GUILLÉN, C. (2015). Engineering students in Spain and Germany-varying and uniform learning strategies [Estudiantes de ingeniería en España y Alemania: estrategias de aprendizaje variadas y uniformes]. In N. Vondrova \& Krainer, K. (Eds.), Proceedings of the gth Congress of European society for Research of Mathematics Education. (2117-2123) Prague: ERME. https://hal.archives-ouvertes.fr/hal01288591/document

GORARD, S. (2003). Quantitative methods in educational research IMétodos cuantitativos en la investigación educativa]. London: Continuum. https://books. google.com.mx/books/about/Quantitative_Methods_in_Social_Science_R. html?id=HSGaUE8alaQC\&redir_esc $=y$

GRIESE, B., LEHMANN, M. Y ROESKEN-WINTER, B. (2015). Refining questionnairebased assessment of STEM students' learning strategies [Refinando la evaluación basada en cuestionarios de las estrategias de aprendizaje de los estudiantes de STEM]. International Journal of STEM Education, 2(1), 12. https:// stemeducationjournal.springeropen.com/articles/10.1186/s40594-015-0025-9

KALDO, I. Y ÕUN, K. (2019). Developing of factor structure for learning strategies of Estonian students in mathematics at the university level IDesarrollo de la estructura factorial para las estrategias de aprendizaje de los estudiantes estonios de matemáticas a nivel universitario]. Problems of Education in the $21^{\text {st }}$ Century, 77(3), 338. http://www.scientiasocialis.tt/pec/node/files/pdf/ vol77/338-348.Kaldo_Vol.77-3_PEC.pdf

KECICl, S. E., \& AYDIN, M. (2019). The adaptation of learning strategies for higher education scale for Turkish context [Adaptación de las estrategias de aprendizaje para la escala de educación superior para el contexto turco]. International Journal of Instruction, 12(1), 1413-1430. https://doi.org/10.29333/iji.2019.12190a

LOCKETT, M., OJEDA, M. Y GILI, A. (2008). Estudio sobre el mejoramiento de los niveles académicos, de estudiantes de la Facultad de Odontologia, analizando estilos y estrategias de aprendizaje. Facultad de Odontología de la Universidad del Nordeste, Argentina.

PINTRICH, P., SMITH, D., GARCIA, T. \& MCKEACHIE, W. (1993). Reliability and predictive validity of the Motivated Strategies for Learning Questionnaire (MSLQ) [Fiabilidad y validez predictiva del Cuestionario de Estrategias Motivadas para el Aprendizaje (MSLQ)]. Educational and Psychological Measurement, 53(3), 801813. http://dx.doi.org/10.1177/0013164493053003024

RODRIGUEZ, G. R. (2018). La reforma de la educación media superior: una compleja transición. Revista de evaluación para docentes y directivos, 9(3), 45-59. 
RUIZ, C. (2015). Confiabilidad. Programa Interinstitucional Doctorado en Educación. Villa, L. L. (2007). La educación media superior ¿igualdad de oportunidades? Revista de la educación superior, 36(141), 93-110. http://www.scielo.org.mx/pdf/ resu/v36n141/v36n141a5.pdf

VIRTANEN, P. Y NEVGI, A. (2010). Disciplinary and gender differences among higher education students in self-regulated learning strategies IDiferencias disciplinarias y de género entre estudiantes de educación superior en estrategias de aprendizaje autorregulado]. Educational Psychology, 30(3), 323347. http://dx.doi.org/10.1080/01443411003606391

WEINSTEIN, C. E. \& PALMER, D. R. (2002). Learning and Study Strategies Inventory (LASSI): User's manual (2nd ed.) IInventario de estrategias de aprendizaje y estudio (LASSI) Manual del usuario]. Clearwater, FL: H\&H Publishing.

WILD, K. P. \& SCHIEFELE, U. (1994). Lernstrategien im studium. Ergebnisse zur Faktorenstruktur und Reliabilität eines neuen Fragebogens [Estrategias de aprendizaje de estudiantes universitarios: estructura factorial y fiabilidad de un nuevo cuestionario]. Zeitschrift für Differentielle und Diagnostische Psychologie, 15. 185-200. https://publishup.uni-potsdam.de/frontdoor/index/index/ docld/3182

WILD, K. P. (2000). Lernstrategien im studium: strukturen und bedingungen [Estrategias de aprendizaje en los estudios: estructuras y condiciones]. Münster: Waxmann. $\quad$ https://Www.fachportal-paedagogik.de/literatur/vollanzeige. html?Fld $=613733$

WILD, K. P. (2005). Individuelle Lernstrategien von Studierenden. Konsequenzen für die Hochschuldidaktik und die Hochschullehre [Estrategias de aprendizaje individuales de los es tudiantes. Consecuencias para la didáctica universitaria y la docencia universitaria]. Beiträge zur Lehrerbildung, 23(2), 191-206. https:// www.pedocs.de/volltexte/2017/13572/pdf/BZL_2005_2_191_206.pdf 